OSFPREPRINTS doi:https://doi.org/10.31219/osf.io/9b2hu. This version posted May 10, 2020. It is made available under a CC-BY-NC-ND 4.0 international license.

"Abouelkhair, Mohamed. 2020. "Targeting the Cd73-adenosine Axis in COVID-19 Immunotherapy." OSF Preprints. May 10. doi:10.31219/osf.io/9b2hu."

\title{
1 Targeting the CD73-adenosine axis in COVID-19 immunotherapy
}

2

3 Mohamed A. Abouelkhair a \#

4

11

12

13

14

15

16

17

18

* Corresponding Author:

Mohamed A. Abouelkhair
aDepartment of Biomedical and Diagnostic Sciences, University of Tennessee College of

Veterinary Medicine, Knoxville, Tennessee, USA

2407 River Dr, Knoxville, TN 37996, USA

Email address: mabouelk@,vols.utk.edu

\section{Keywords: COVID-19, Immunotherapy, CD73, Adenosine, $A_{2 A} R$ antagonist}


OSFPREPRINTS doi:https://doi.org/10.31219/osf.io/9b2hu. This version posted May 10, 2020. It is made available under a CC-BY-NC-ND 4.0 international license.

"Abouelkhair, Mohamed. 2020. "Targeting the Cd73-adenosine Axis in COVID-19 Immunotherapy." OSF Preprints. May 10. doi:10.31219/osf.io/9b2hu."

\section{Abstract}

26 The most serious health issue today is the rapid outbreak of Coronavirus Disease 2019 (COVID-

2719 or SARS-CoV-2). It is now a global pandemic and is a huge concern for public health. So far,

28 more than 3,500,000 confirmed cases were diagnosed in nearly 212 countries and territories

29 around the world and 2 international conveyances, causing globally over 250,000 deaths.

30 Epidemiology, risk factors, and clinical characteristics of COVID-19 patients have been

31 identified but the factors influencing the immune system against COVID-19 have not been well

32 established. Upon infection or cell damage, high amounts of adenosine triphosphate (ATP) are

33 released from damaged cells, which serve as mediators of inflammation through purinergic

34 cell surface receptor signaling. As a protective mechanism to prevent excessive damage to host

35 tissue, adenosine counteracts ATP's effects by adenosine receptor stimulation to suppress the

36 pro-inflammatory response. Currently, the adenosine pathway is seen as a major obstacle to the

37 efficacy of immune therapies and is an important therapeutic target for cancer and microbial

38 infections. Pharmacologic inhibitors or antibodies specific to adenosine pathway components or

39 adenosine receptors in microbial and tumor therapy have shown efficacy in pre-clinical studies

40 and are entering the clinical arena. In this review, I propose a novel hypothesis explaining the

41 potential for improving the efficiency of innate and adaptive immune systems by co-inhibition of

42 CD73 and $\mathrm{A}_{2 \mathrm{~A}} \mathrm{R}$ adenosine Signaling for COVID-19 prevention and control.

\section{Introduction}

44 In December 2019, the novel coronavirus (COVID-19 or SARS-CoV-2) emerged as part of a 45 major respiratory disease outbreak centered on Hubei Province, China (Gralinski and 
OSFPREPRINTS doi:https://doi.org/10.31219/osf.io/9b2hu. This version posted May 10, 2020. It is made available under a CC-BY-NC-ND 4.0 international license.

"Abouelkhair, Mohamed. 2020. "Targeting the Cd73-adenosine Axis in COVID-19 Immunotherapy." OSF Preprints. May 10. doi:10.31219/osf.io/9b2hu."

46 Menachery, 2020). It is now a global pandemic and is a huge concern for public health. So far,

47 more than 3,500,000 confirmed cases were diagnosed in nearly 212 countries and territories

48 around the world and 2 international conveyances, causing globally over 250,000 deaths

49 (Worldometer, 2020, May 02). Taxonomically, SARS-CoV-2 is a Betacoronavirus (lineage B)

50 closely linked to SARS-CoV and SARS-related bat coronaviruses (Jaimes et al., 2020).

51 Thereafter, beta coronaviruses from pangolins were described as close relatives to SARS-CoV-2

52 (Jaimes et al., 2020).

53 Owing to the genomic similarity of $79 \%$ with SARS-CoV, almost the same immune system

54 response is predicted to occur against COVID-19. With the accumulated clinical and

55 experimental data on SARS-CoV, it is possible to hypothesize and even predict how the host

56 immune system can deal with this particular virus and how the virus can avoid such host

57 reactions (Lu et al., 2020; Taghizadeh-Hesary and Akbari, 2020). The most common symptoms

58 of COVID-19 are fever, fatigue, and respiratory symptoms such as cough, sore throat, and acute

59 respiratory distress syndrome (ARDS). Although diarrhea was reported in approximately 20-

$6025 \%$ of patients with SARS-CoV, intestinal symptoms were rarely reported in patients with

61 COVID-19 (Huang et al., 2020; Wu et al., 2020).

62 Both the innate and adaptive immune systems are involved in response to SARS-CoV. In order

63 to defeat the immune response, SARS-CoV applies different mechanisms. One of these

64 mechanisms is through inhibition of interferon type 1 (IFN-1) expression and signaling (de Wit

65 et al., 2016; Kindler and Thiel, 2016).

66 IFNs can be divided into three subtypes: type I ( $\alpha$ and $\beta)$, type II $(\gamma)$, and type III $(\lambda)$ (Parkin and

67 Cohen, 2001; Akira et al., 2006; Zhang et al., 2017b). Type I IFNs are evolutionarily preserved 
OSFPREPRINTS doi:https://doi.org/10.31219/osf.io/9b2hu. This version posted May 10, 2020. It is made available under a CC-BY-NC-ND 4.0 international license.

"Abouelkhair, Mohamed. 2020. "Targeting the Cd73-adenosine Axis in COVID-19 Immunotherapy." OSF Preprints. May 10. doi:10.31219/osf.io/9b2hu."

68 and consist of multiple IFN- $\alpha$ subtypes (14 in human, 11 in mouse) and single subtypes $\beta, \varepsilon, \tau$,

$69 \omega, \zeta$, and $\kappa$, all of which are essential in the fight against RNA and DNA virus infection (Chen et

70 al., 2004). IFN-I are all recognized by interferon- $\alpha / \beta$ receptor (IFNAR), which results in the

71 activation of multiple signaling effectors and antiviral response pathways that coordinate

72 invoking gene and protein regulation including IFN-stimulated protein of $15 \mathrm{kDa}$ (ISG15), the

73 myxovirus resistance $1(\mathrm{Mx} 1)$, ribonuclease $\mathrm{L}(\mathrm{RNaseL})$ and protein kinase $\mathrm{R}$ in the target cells,

74 all of them exert potential direct antiviral effects (Zhao et al., 2005; Sadler and Williams, 2008).

75 In the meantime, inducible type I IFN plays a key role in antiviral immune responses by inducing

76 cellular resistance to viral infection and promoting apoptosis of virus-infected cells (Zhao et al.,

77 2005; Taghizadeh-Hesary and Akbari, 2020).

\section{ATP limits viral replication by facilitating IF $-\beta$ production and}

\section{9 signaling}

80 The various functions of living organisms are regulated by extracellular signaling of purines and

81 pyrimidine derivatives, including adenosine triphosphate (ATP) (Abbracchio and Burnstock,

82 1998), adenosine diphosphate (ADP) (Burnstock, 2006) and adenosine (Zimmermann, 1992).

83 Such molecules are produced via the release from cells or the metabolic hydrolysis of their

84 precursors in the extracellular space. The physiological roles of these nucleotides and

85 nucleosides had not been investigated until the 1970s. The focus of the research was limited to

86 the synthesis of ATP and its energy role.

87 ATP plays a primary role in stimulating the inflammatory response via the purinergic pathway

88 using P2Y receptors (P2YRs) (Jacob et al., 2013). ATP can be highly released by virus-infected

89 cells during the early stages of infection, which is different from pathogen-induced cytokines 
OSFPREPRINTS doi:https://doi.org/10.31219/osf.io/9b2hu. This version posted May 10, 2020. It is made available under a CC-BY-NC-ND 4.0 international license.

"Abouelkhair, Mohamed. 2020. "Targeting the Cd73-adenosine Axis in COVID-19 Immunotherapy." OSF Preprints. May 10. doi:10.31219/osf.io/9b2hu."

(Taghizadeh-Hesary and Akbari, 2020). Thus, the released ATP could serve as a danger signal to

91 alarm the immune system to fight against invading pathogens.

92 SARS-CoV impairs the rapid increase of the IFN-I. As a result, the so-called "initial alarm" of

93 the innate immune system is deactivated by unknown mechanisms (Taghizadeh-Hesary and

94 Akbari, 2020). This promotes coronavirus replication (Zhang et al., 2017a). Such rapid SARS-

$95 \mathrm{CoV}$ replication kinetics and relative delay in IFN-I signaling orchestrate induction of an

96 inappropriate inflammatory response during SARS-CoV infection, extensive vascular leakage,

97 impaired virus-specific T-cell responses and consequent immunopathology of the lungs in

98 infected BALB/c mice (Channappanavar et al., 2016). Such results are similar findings in SARS

99 patients (Nicholls et al., 2003; Channappanavar et al., 2016). Therefore, not only the magnitude

100 of the response but also its timing may seem to play a crucial role in the infection with

101 coronavirus (Figure 1).

102 Zhang et al. have shown that improving extracellular ATP can reverse this cycle (Zhang et al.,

103 2017a) by facilitating the secretion of IFN through the signaling pathway P38/JNK/ATF-2

104 (Zhang et al., 2017a). Consequently, ATP-depleted cells are more vulnerable to viral infections

105 such as SAR-CoV and probably COVID-19. (Johnson et al., 2019).

106 Fortunately, Kumari G et al. found that SARS-CoV-2 is more susceptible to IFN-I pretreatment

107 than SARS-CoV and type I IFN can be useful for SARS-CoV-2 treatment if appropriate

108 parameters are determined (Lokugamage et al., 2020).

109 Fundamental changes occur in the immune cells after IFN-I secretion, which turns them into the

110 so-called antiviral state (Parkin and Cohen, 2001). Janus kinase (JAK)-signal transducer and

111 activator of transcription (STAT) pathway is one of the signaling pathways that participate in this 
OSFPREPRINTS doi:https://doi.org/10.31219/osf.io/9b2hu. This version posted May 10, 2020. It is made available under a CC-BY-NC-ND 4.0 international license.

"Abouelkhair, Mohamed. 2020. "Targeting the Cd73-adenosine Axis in COVID-19 Immunotherapy." OSF Preprints. May 10. doi:10.31219/osf.io/9b2hu."

112 process (Renauld, 2003; Seif et al., 2017). JAKs are ATP-dependent enzymes which are bound

113 to cytoplasmic regions of type I and II cytokine receptors. Upon IFN-I stimulation, JAKs are

114 activated and phosphorylate STATs which results in dimerization and translocation of STATs to

115 the nucleus (Seif et al., 2017). Cellular ATP depletion obviously interferes with IFN-I signaling

116 and thus impairs the transformation into "antiviral state" (Taghizadeh-Hesary and Akbari, 2020).

CD73 and Adenosine Receptor Activity Promote Immunosuppression

119 Nucleotides and adenosine are metabolized by various enzymes in the extracellular space. These

120 nucleotide-metabolizing enzymes, collectively called ecto-nucleotidases, have potential as drug

121 targets (Zimmermann et al., 2012). Nucleotides such as adenosine triphosphate (ATP) and

122 adenosine diphosphate (ADP) are extruded from damaged and necrotic cells at inflammation

123 sites and hydrolyzed by CD39 into adenosine monophosphate (AMP) (Perrot et al., 2019). AMP

124 is then hydrolyzed to adenosine by CD73 (Perrot et al., 2019). Successive ATP processing by

125 CD73 and CD39 ectonucleotidases decreases cellular ATP levels and can rapidly increase

126 adenosine from a low homeostatic level (20-200 nM) to as much as 1,000-10,000 nM (Abdel-

127 Magid, 2017). These elevated concentrations of adenosine exert immunosuppressive action

128 through adenosine $\mathrm{A}_{2 \mathrm{~A}}$ and $\mathrm{A}_{2 \mathrm{~B}}$ receptors on infiltrating lymphocytes, shielding the cells from

129 excessive inflammatory reactions and thus providing a self-limiting mechanism to resolve the

130 immune response (Ohta and Sitkovsky, 2001).

131 Nonetheless, crucial mechanisms underlying lung injury and dysfunction in COVID-19 or any

132 other pulmonary viral infection are currently poorly described. Influenza virus infection of

$133 \mathrm{BALB} / \mathrm{c}$ mice induces increased channel-mediated release of nucleotides UTP and ATP into

134 bronchoalveolar lining fluid (BALF) (Aeffner et al., 2012), which contributes to lung edema and 
OSFPREPRINTS doi:https://doi.org/10.31219/osf.io/9b2hu. This version posted May 10, 2020. It is made available under a CC-BY-NC-ND 4.0 international license.

"Abouelkhair, Mohamed. 2020. "Targeting the Cd73-adenosine Axis in COVID-19 Immunotherapy." OSF Preprints. May 10. doi:10.31219/osf.io/9b2hu."

135 hypoxemia. It was hypothesized that influenza induces de novo ATP synthesis and release from

136 infected Alveolar epithelial type II (ATII) cells. The released ATP in BALF could be rapidly

137 metabolized to adenosine at an accelerated rate (due to increased ectonucleotidase CD73

138 activity), which plays a pivotal role in influenza lung injury due to its impact on adenosine

139 receptor (Wolk et al., 2008).

140 Targeting the CD73-adenosine axis may potentially help for the

141 prevention and control of COVID-19

142 Pharmacologic inhibitors or antibodies to CD39 and CD73 ectonucleotidases may potentially

143 have preventive effects through the protection of extracellular ATP from hydrolysis and

144 production of immunosuppressive molecule, adenosine, and maintaining the ATP level for

145 activating the initial IFN-I secretion and signaling as "initial alarm" of the innate immune system

146 (Figure 2).

147 In the last decade, the potential of CD73 and $\mathrm{A}_{2 \mathrm{~A}} \mathrm{R}$ antagonist as immunotherapy targets have

148 rapidly increased (Willingham et al.; Siu et al., 2018; Powderly et al., 2019; Harvey et al., 2020).

149 Adenosine $\mathrm{A}_{2 \mathrm{~A}}$ receptor antagonist, for example istradefylline and Ciforadenant, binds to

150 adenosine $\mathrm{A}_{2 \mathrm{~A}}$ receptors on the surface of the immune cells such as T-lymphocytes, natural killer

151 cells (NK), macrophages, and dendritic cells (DCs) (Willingham et al.; Antonioli et al., 2008).

$152 \mathrm{~A}_{2 \mathrm{~A}}$ receptor anatagonist prevents adenosine from interacting with the $\mathrm{A}_{2 \mathrm{~A}}$ receptors of these

153 primary immune surveillance cells, thus eliminating the immunosuppression. Ciforadenant

154 (formerly CPI-444), an oral $\mathrm{A}_{2 \mathrm{~A}} \mathrm{R}$ antagonist, suppresses the expression of several checkpoint

155 pathways on CD8 + effector T cells and CD4 + FoxP3 + Tregs and also have profound effects in 
OSFPREPRINTS doi:https://doi.org/10.31219/osf.io/9b2hu. This version posted May 10, 2020. It is made available under a CC-BY-NC-ND 4.0 international license.

"Abouelkhair, Mohamed. 2020. "Targeting the Cd73-adenosine Axis in COVID-19 Immunotherapy." OSF Preprints. May 10. doi:10.31219/osf.io/9b2hu."

156 restoring antitumor immunity at draining lymph nodes by decreasing the expression of

157 programmed cell death (PD-1) and lymphocyte-activation gene 3 (LAG-3) (Leone et al., 2015).

158 Monoclonal anti-CD73 antibody BMS-986179 displayed possible immunomodulatory and

159 antineoplastic activity (Siu et al., 2018). Anti-CD73 monoclonal antibody targets and binds to

160 CD73 upon administration, leading to clustering and internalization of CD73 (Terp et al., 2013).

161 This prevents CD73-mediated conversion of extracellular adenosine monophosphate (AMP) to

162 adenosine and decreases free adenosine which blocks adenosine-mediated suppression of

163 lymphocyte activity and increases CD8-positive cell function. This also stimulates macrophages,

164 suppressing both myeloid-derived suppressor cells (MDSCs) and regulatory T lymphocytes.

166 Conclusions

167 Given these considerations, a novel hypothesis is proposed, explaining the potential for

168 improving the efficiency of innate and adaptive immune response for COVID-19 prevention and

169 control by blocking the production of adenosine via CD73 and signaling via $A_{2 A} R$. The cellular

170 ATP level can theoretically be considered a key component in COVID-19 infectivity and

171 prognosis. Enhancing cellular ATP, through targeting CD73-adenosine axis is predicted to

172 strengthen both innate and cell-mediated immune systems. The therapeutic gain of targeting both

173 components of the adenosine pathway is much higher than one because it inhibits the

174 compensatory response of $\mathrm{A}_{2 \mathrm{~A}} \mathrm{R}$ blockade to increase CD73. This indicates the potential benefits

175 of integrating adenosine-related therapeutic approaches with existing clinically approved

176 antiviral drugs. 
OSFPREPRINTS doi:https://doi.org/10.31219/osf.io/9b2hu. This version posted May 10, 2020. It is made available under a CC-BY-NC-ND 4.0 international license.

"Abouelkhair, Mohamed. 2020. "Targeting the Cd73-adenosine Axis in COVID-19 Immunotherapy." OSF Preprints. May 10. doi:10.31219/osf.io/9b2hu."

\section{Reference}

Abbracchio, M.P., and Burnstock, G. (1998). Purinergic signalling: pathophysiological roles. Jpn J Pharmacol 78(2), 113-145. doi: 10.1254/jjp.78.113.

Abdel-Magid, A.F. (2017). Inhibitors of CD73 May Provide a Treatment for Cancer and Autoimmune Diseases. ACS medicinal chemistry letters 8(8), 781-782. doi: 10.1021/acsmedchemlett.7b00255.

Aeffner, F., Bratasz, A., Flano, E., Powell, K.A., and Davis, I.C. (2012). Postinfection A77-1726 treatment improves cardiopulmonary function in H1N1 influenza-infected mice. Am J Respir Cell Mol Biol 47(4), 543-551. doi: 10.1165/rcmb.2012-01120C.

Akira, S., Uematsu, S., and Takeuchi, O. (2006). Pathogen recognition and innate immunity. Cell 124(4), 783-801. doi: 10.1016/j.cell.2006.02.015.

Antonioli, L., Fornai, M., Colucci, R., Ghisu, N., Tuccori, M., Del Tacca, M., et al. (2008). Regulation of enteric functions by adenosine: pathophysiological and pharmacological implications. Pharmacol Ther 120(3), 233-253. doi: 10.1016/j.pharmthera.2008.08.010.

Burnstock, G. (2006). Purinergic signalling. Br J Pharmacol 147 Suppl 1, S172-181. doi: 10.1038/sj.bjp.0706429.

Channappanavar, R., Fehr, A.R., Vijay, R., Mack, M., Zhao, J., Meyerholz, D.K., et al. (2016). Dysregulated Type I Interferon and Inflammatory Monocyte-Macrophage Responses Cause Lethal Pneumonia in SARS-CoV-Infected Mice. Cell host \& microbe 19(2), 181-193. doi: 10.1016/j.chom.2016.01.007.

Chen, J., Baig, E., and Fish, E.N. (2004). Diversity and relatedness among the type I interferons. J Interferon Cytokine Res 24(12), 687-698. doi: 10.1089/jir.2004.24.687.

de Wit, E., van Doremalen, N., Falzarano, D., and Munster, V.J. (2016). SARS and MERS: recent insights into emerging coronaviruses. Nat Rev Microbiol 14(8), 523-534. doi: 10.1038/nrmicro.2016.81.

Gralinski, L.E., and Menachery, V.D. (2020). Return of the Coronavirus: 2019-nCoV. Viruses 12(2). doi: 10.3390/v12020135.

Harvey, J.B., Phan, L.H., Villarreal, O.E., and Bowser, J.L. (2020). CD73's Potential as an Immunotherapy Target in Gastrointestinal Cancers. Frontiers in immunology 11, 508508. doi: 10.3389/fimmu.2020.00508.

Huang, C., Wang, Y., Li, X., Ren, L., Zhao, J., Hu, Y., et al. (2020). Clinical features of patients infected with 2019 novel coronavirus in Wuhan, China. Lancet 395(10223), 497-506. doi: 10.1016/S0140-6736(20)30183-5.

Jacob, F., Pérez Novo, C., Bachert, C., and Van Crombruggen, K. (2013). Purinergic signaling in inflammatory cells: P2 receptor expression, functional effects, and modulation of inflammatory responses. Purinergic signalling 9(3), 285-306. doi: 10.1007/s11302-0139357-4.

Jaimes, J.A., Andre, N.M., Chappie, J.S., Millet, J.K., and Whittaker, G.R. (2020). Phylogenetic Analysis and Structural Modeling of SARS-CoV-2 Spike Protein Reveals an Evolutionary 
OSFPREPRINTS doi:https://doi.org/10.31219/osf.io/9b2hu. This version posted May 10, 2020. It is made available under a CC-BY-NC-ND 4.0 international license.

"Abouelkhair, Mohamed. 2020. "Targeting the Cd73-adenosine Axis in COVID-19 Immunotherapy." OSF Preprints. May 10. doi:10.31219/osf.io/9b2hu."

Distinct and Proteolytically Sensitive Activation Loop. J Mol Biol. doi: 10.1016/j.jmb.2020.04.009.

Johnson, T.A., Jinnah, H.A., and Kamatani, N. (2019). Shortage of Cellular ATP as a Cause of Diseases and Strategies to Enhance ATP. Front Pharmacol 10, 98. doi: 10.3389/fphar.2019.00098.

Kindler, E., and Thiel, V. (2016). SARS-CoV and IFN: Too Little, Too Late. Cell Host Microbe 19(2), 139-141. doi: 10.1016/j.chom.2016.01.012.

Leone, R.D., Lo, Y.-C., and Powell, J.D. (2015). A2aR antagonists: Next generation checkpoint blockade for cancer immunotherapy. Computational and structural biotechnology journal 13, 265-272. doi: 10.1016/j.csbj.2015.03.008.

Lokugamage, K.G., Hage, A., Schindewolf, C., Rajsbaum, R., and Menachery, V.D. (2020). SARSCoV-2 is sensitive to type I interferon pretreatment. bioRxiv, 2020.2003.2007.982264. doi: 10.1101/2020.03.07.982264.

Lu, R., Zhao, X., Li, J., Niu, P., Yang, B., Wu, H., et al. (2020). Genomic characterisation and epidemiology of 2019 novel coronavirus: implications for virus origins and receptor binding. The Lancet 395(10224), 565-574.

Nicholls, J.M., Poon, L.L., Lee, K.C., Ng, W.F., Lai, S.T., Leung, C.Y., et al. (2003). Lung pathology of fatal severe acute respiratory syndrome. Lancet 361(9371), 1773-1778. doi: 10.1016/s0140-6736(03)13413-7.

Ohta, A., and Sitkovsky, M. (2001). Role of G-protein-coupled adenosine receptors in downregulation of inflammation and protection from tissue damage. Nature 414(6866), 916-920. doi: 10.1038/414916a.

Parkin, J., and Cohen, B. (2001). An overview of the immune system. Lancet 357(9270), 17771789. doi: 10.1016/S0140-6736(00)04904-7.

Perrot, I., Michaud, H.-A., Giraudon-Paoli, M., Augier, S., Docquier, A., Gros, L., et al. (2019). Blocking Antibodies Targeting the CD39/CD73 Immunosuppressive Pathway Unleash Immune Responses in Combination Cancer Therapies. Cell Reports 27(8), 24112425.e2419. doi: https://doi.org/10.1016/j.celrep.2019.04.091.

Powderly, J.D., de Souza, P.L., Gutierrez, R., Horvath, L., Seitz, L., Ashok, D., et al. (2019). AB928, a novel dual adenosine receptor antagonist, combined with chemotherapy or AB122 (anti-PD-1) in patients (pts) with advanced tumors: Preliminary results from ongoing phase I studies. Journal of Clinical Oncology 37(15_suppl), 2604-2604. doi: 10.1200/JCO.2019.37.15_suppl.2604.

Renauld, J.C. (2003). Class II cytokine receptors and their ligands: key antiviral and inflammatory modulators. Nat Rev Immunol 3(8), 667-676. doi: 10.1038/nri1153.

Sadler, A.J., and Williams, B.R.G. (2008). Interferon-inducible antiviral effectors. Nature reviews. Immunology 8(7), 559-568. doi: 10.1038/nri2314.

Seif, F., Khoshmirsafa, M., Aazami, H., Mohsenzadegan, M., Sedighi, G., and Bahar, M. (2017). The role of JAK-STAT signaling pathway and its regulators in the fate of T helper cells. Cell Communication and Signaling 15(1), 23. doi: 10.1186/s12964-017-0177-y.

Siu, L.L., Burris, H., Le, D.T., Hollebecque, A., Steeghs, N., Delord, J.-P., et al. (2018). Abstract CT180: Preliminary phase 1 profile of BMS-986179, an anti-CD73 antibody, in 
OSFPREPRINTS doi:https://doi.org/10.31219/osf.io/9b2hu. This version posted May 10, 2020. It is made available under a CC-BY-NC-ND 4.0 international license.

"Abouelkhair, Mohamed. 2020. "Targeting the Cd73-adenosine Axis in COVID-19 Immunotherapy." OSF Preprints. May 10. doi:10.31219/osf.io/9b2hu." combination with nivolumab in patients with advanced solid tumors. Cancer Research 78(13 Supplement), CT180. doi: 10.1158/1538-7445.AM2018-CT180.

Taghizadeh-Hesary, F., and Akbari, H. (2020). The Powerful Immune System Against Powerful COVID-19: A Hypothesis. Medical Hypotheses, 109762. doi: https://doi.org/10.1016/j.mehy.2020.109762.

Terp, M.G., Olesen, K.A., Arnspang, E.C., Lund, R.R., Lagerholm, B.C., Ditzel, H.J., et al. (2013). Anti-human CD73 monoclonal antibody inhibits metastasis formation in human breast cancer by inducing clustering and internalization of CD73 expressed on the surface of cancer cells. J Immunol 191(8), 4165-4173. doi: 10.4049/jimmunol.1301274.

Willingham, S.B., Ho, P.Y., Hotson, A., Hill, C., Piccione, E.C., Hsieh, J.A.-O., et al. A2AR Antagonism with CPI-444 Induces Antitumor Responses and Augments Efficacy to AntiPD-(L)1 and Anti-CTLA-4 in Preclinical Models. (2326-6074 (Electronic)).

Wolk, K.E., Lazarowski, E.R., Traylor, Z.P., Yu, E.N., Jewell, N.A., Durbin, R.K., et al. (2008). Influenza A virus inhibits alveolar fluid clearance in BALB/c mice. Am J Respir Crit Care Med 178(9), 969-976. doi: 10.1164/rccm.200803-4550C.

Worldometer (2020, May 02). Covid-19 coronavirus pandemic.

Wu, F., Zhao, S., Yu, B., Chen, Y.-M., Wang, W., Song, Z.-G., et al. (2020). Author Correction: A new coronavirus associated with human respiratory disease in China. Nature 580(7803), E7-E7. doi: 10.1038/s41586-020-2202-3.

Zhang, C., He, H., Wang, L., Zhang, N., Huang, H., Xiong, Q., et al. (2017a). Virus-Triggered ATP Release Limits Viral Replication through Facilitating IFN-beta Production in a P2X7Dependent Manner. J Immunol 199(4), 1372-1381. doi: 10.4049/jimmunol.1700187.

Zhang, C., He, H., Wang, L., Zhang, N., Huang, H., Xiong, Q., et al. (2017b). Virus-Triggered ATP Release Limits Viral Replication through Facilitating IFN- $\beta$ Production in a P2X7Dependent Manner. The Journal of Immunology 199(4), 1372. doi: 10.4049/jimmunol.1700187.

Zhao, C., Denison, C., Huibregtse, J.M., Gygi, S., and Krug, R.M. (2005). Human ISG15 conjugation targets both IFN-induced and constitutively expressed proteins functioning in diverse cellular pathways. Proc Natl Acad Sci U S A 102(29), 10200-10205. doi: 10.1073/pnas.0504754102.

Zimmermann, H. (1992). 5'-Nucleotidase: molecular structure and functional aspects. Biochem J 285 ( Pt 2), 345-365. doi: 10.1042/bj2850345.

Zimmermann, H., Zebisch, M., and Strater, N. (2012). Cellular function and molecular structure of ecto-nucleotidases. Purinergic Signal 8(3), 437-502. doi: 10.1007/s11302-012-9309-4. 
OSFPREPRINTS doi:https://doi.org/10.31219/osf.io/9b2hu. This version posted May 10, 2020. It is made available under a CC-BY-NC-ND 4.0 international license.

"Abouelkhair, Mohamed. 2020. "Targeting the Cd73-adenosine Axis in COVID-19 Immunotherapy." OSF Preprints. May 10. doi:10.31219/osf.io/9b2hu."

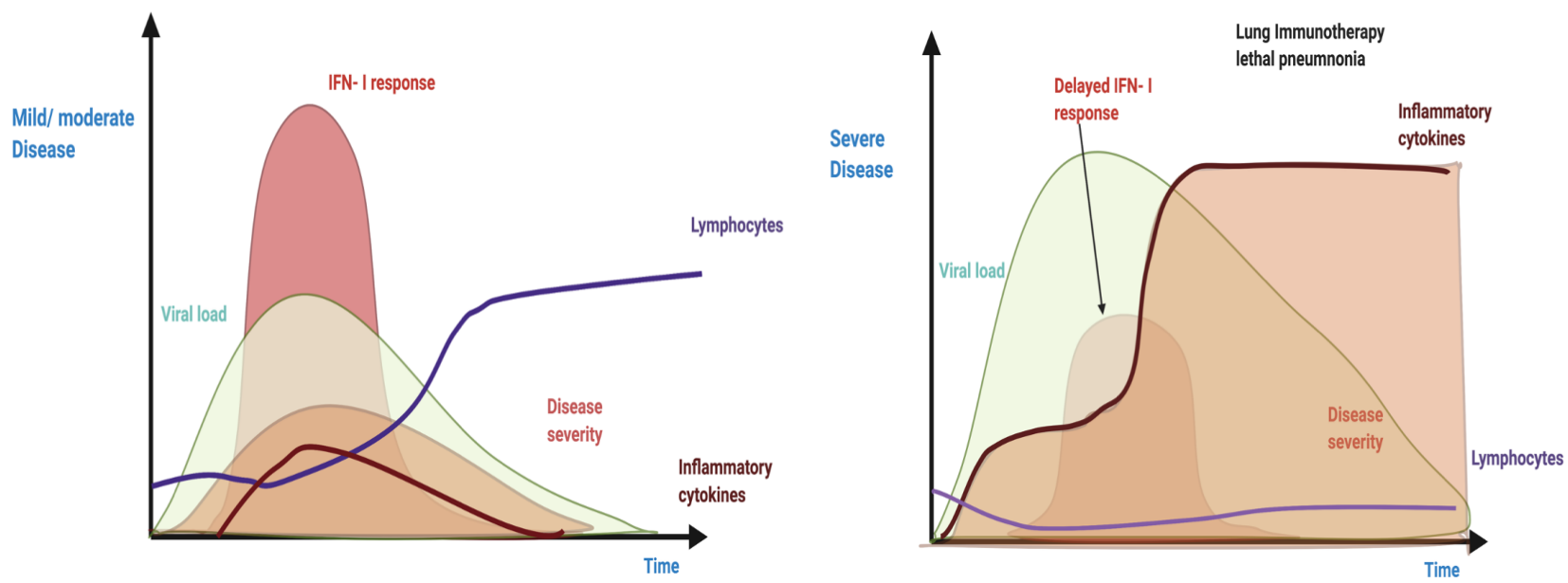

301 Figure 1: Kinetics and the magnitude of the IFN-1 response are crucial in infection with

302 coronavirus. Relative delay in IFN-I signaling orchestrates the induction of an inappropriate

303 inflammatory response and consequent lung immunopathology. 
OSFPREPRINTS doi:https://doi.org/10.31219/osf.io/9b2hu. This version posted May 10, 2020. It is made available under a CC-BY-NC-ND 4.0 international license.

"Abouelkhair, Mohamed. 2020. "Targeting the Cd73-adenosine Axis in COVID-19 Immunotherapy." OSF Preprints. May 10. doi:10.31219/osf.io/9b2hu."

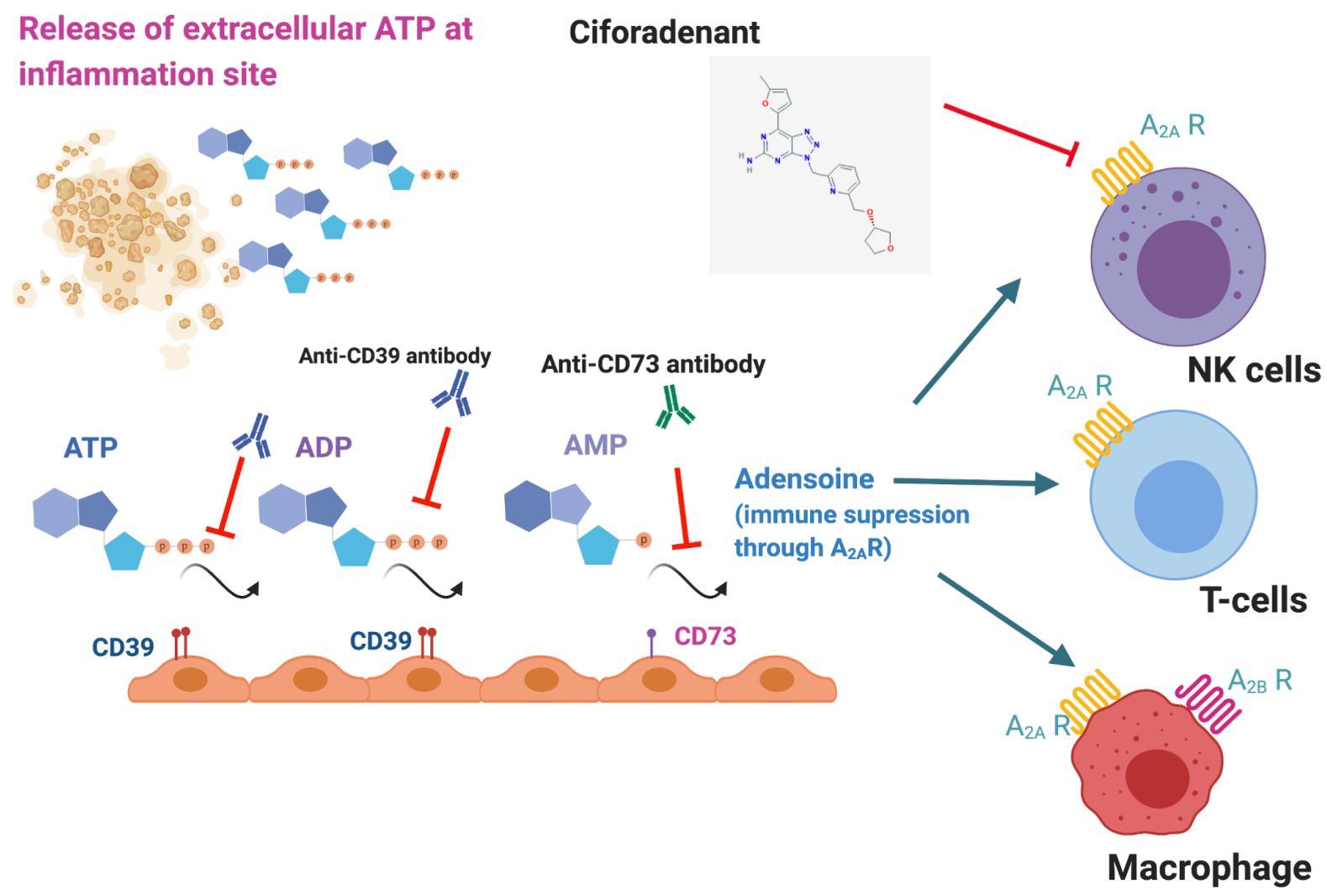

318 Figure 2: Targeting the CD73-adenosine axis by using anti-CD73 monoclonal antibodies and

$319 \quad \mathrm{~A}_{2 \mathrm{~A}} \mathrm{R}$ receptor antagonist. 